\title{
BMJ Open Medicoeconomic analysis of lobectomy using thoracoscopy versus thoracotomy for lung cancer: a study protocol for a multicentre randomised controlled trial (Lungsco01)
}

\author{
Pierre-Benoit Pagès, ${ }^{1}$ Halim Abou Hanna, ${ }^{1}$ Anne-Claire Bertaux, ${ }^{2}$ \\ Ludwig Serge Serge Aho, ${ }^{3}$ Pierre Magdaleinat, ${ }^{4}$ Jean-Marc Baste, ${ }^{5}$ Marc Filaire, ${ }^{6}$ \\ Richard de Latour, ${ }^{7}$ Jalal Assouad, ${ }^{8}$ François Tronc, ${ }^{9}$ Christophe Jayle, ${ }^{10}$ \\ Jérome Mouroux, ${ }^{11}$ Pascal-Alexandre Thomas, ${ }^{12}$ Pierre-Emmanuel Falcoz, ${ }^{13}$ \\ Charles-Henri Marty-Ané, ${ }^{14}$ Alain Bernard ${ }^{1}$
}

To cite: Pagès $P-B, A b o u$ Hanna $\mathrm{H}$, Bertaux A-C, et al. Medicoeconomic analysis of lobectomy using thoracoscopy versus thoracotomy for lung cancer: a study protocol for a multicentre randomised controlled trial (Lungsc001). BMJ Open 2017;7:e012963. doi:10.1136/ bmjopen-2016-012963

- Prepublication history and additional material are available. To view these files please visit the journal online (http://dx.doi. org/10.1136/bmjopen-201602963).

Received 7 June 2016 Revised 29 November 2016 Accepted 6 December 2016

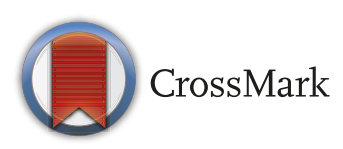

For numbered affiliations see end of article.

Correspondence to Dr Pierre-Benoit Pagès; pierrebenoit.pages@chu-dijon.fr

\section{ABSTRACT}

Introduction In the last decade, video-assisted thoracoscopic surgery (VATS) lobectomy for non-small cell lung cancer (NSCLC) has had a major effect on thoracic surgery. Retrospective series have reported benefits of VATS when compared with open thoracotomy in terms of postoperative pain, postoperative complications and length of hospital stay. However, no large randomised control trial has been conducted to assess the reality of the potential benefits of VATS lobectomy or its medicoeconomic impact.

Methods and analysis The French National Institute of Health funded Lungsco01 to determine whether VATS for lobectomy is superior to open thoracotomy for the treatment of NSCLC in terms of economic cost to society. This trial will also include an analysis of postoperative outcomes, the length of hospital stay, the quality of life, long-term survival and locoregional recurrence. The study design is a two-arm parallel randomised controlled trial comparing VATS lobectomy with lobectomy using thoracotomy for the treatment of NSCLC. Patients will be eligible if they have proven or suspected lung cancer which could be treated by lobectomy. Patients will be randomised via an independent service. All patients will be monitored according to standard thoracic surgical practices. All patients will be evaluated at day 1 , day 30 , month 3 , month 6 , month 12 and then every year for 2 years thereafter. The recruitment target is 600 patients.

Ethics and dissemination The protocol has been approved by the French National Research Ethics Committee (CPP Est I: 09/06/2015) and the French Medicines Agency (09/06/2015). Results will be presented at national and international meetings and conferences and published in peer-reviewed journals.

Trial registration number NCT02502318.

\section{INTRODUCTION}

\section{Background and rationale}

In 2012, in France, the incidence and mortality rates for lung cancer were, respectively, 51.7
Strengths and limitations of this study

- To date, all of the publications dealing with videoassisted thoracic surgery (VATS) lobectomy have been retrospective observational studies based on propensity scores in order to reduce bias; however, randomised controlled trials (RCTs) are the only experimental designs to provide optimal control of bias.

- This trial will be the first prospective multicentre RCT to assess the medicoeconomic impact of VATS lobectomy when compared with open thoracotomy for the management of non-small cell lung cancer (NSCLC) from the societal perspective.

- This trial is expected to determine whether VATS lobectomy provides benefits in terms of postoperative complications, quality of life, postoperative mortality, length of hospital stay and overall survival when compared with open thoracotomy for the management of NSCLC.

- Validation of the judgement criteria will be performed blindly by an independent adjudication committee that includes three thoracic surgeons.

- The limitations are inherent to RCTs: they concern the extrapolation of the results in centres which do not have the same expertise as the trial participating centres, as well as the estimation of overall survival and disease-free survival which are secondary assessment criteria.

and 37.0 per 100000 person-years in men and 18.6 and 12.9 per 100000 person-years in women, and around 30000 people died. ${ }^{1}$ No randomised controlled trial (RCT) has compared surgery alone with radiotherapy or chemotherapy alone, or ablative therapies in otherwise healthy patients with stage I and II non-small cell lung cancer (NSCLC). 
The argument for surgery comes primarily from consistent data from retrospective surgical series, databases and registries showing higher survival rates after surgery than after other treatment modalities. ${ }^{2}$ The type of lung resection is quite well codified (lobectomy or pneumonectomy) depending on the size and location of the tumour. Today, the conventional approach for lobectomy is thoracotomy, which could be posterolateral or lateral thoracotomy. ${ }^{2}$ Currently, there is no scientific evidence to say that one type of thoracotomy is better than the other. Lobectomy is a major surgery for patients and morbidity is high. Respiratory complications are the main complications after lobectomies performed by thoracotomy, affecting around $12 \%$ of patients according to the results from the National North-American Thoracic Surgery database. ${ }^{3}$ These results are confirmed by data from the French National Society of Thoracic and Cardiovascular Surgery database, which reported a rate of respiratory complications of around $15 \% .{ }^{4}$ Respiratory complications have a major impact on the length of stay in intensive care units (ICUs) and hospitals and, in the more severe cases, could lead to acute respiratory distress syndrome (ARDS) and death. ${ }^{5}$

In the last decade, video-assisted thoracoscopic surgery (VATS) lobectomy for NSCLC has had a major effect on thoracic surgery. ${ }^{6}$ In France, the use of VATS lobectomy increased from $1.3 \%$ of lobectomies in 2003 to $13 \%$ in $2012 .{ }^{6}$ The major benefit of this minimally invasive procedure is to reduce postoperative pain and improve quality of life. ${ }^{7}$

A recent RCT involving 206 patients and comparing VATS with open thoracotomy showed a benefit of VATS regarding postoperative pain and quality of life during the first year of follow-up. ${ }^{7}$ The authors highlighted a major decrease in the number of episodes of moderate-to-severe pain and an increase in quality of life scores during the 52 weeks of follow-up. Moreover, recent meta-analyses have highlighted a decrease in the length of hospital stay and a decrease in postoperative complications, such as atelectasis or pneumonia. ${ }^{8-15}$ To date, only three RCTs comparing VATS lobectomy with open thoracotomy for the treatment of lung cancer, in terms of complications and overall survival, have been published in English. ${ }^{16-18}$ Moreover, these trials included fewer than 100 patients each and were all conducted in a single centre. Two of these trials were not defined by a single principal judgement criterion but several heterogeneous criteria, making it difficult to interpret and extrapolate the results. ${ }^{16}{ }^{19}$ Another trial had a judgement criterion which was not clinically relevant. ${ }^{20}$

In a recent publication from the Surveillance, Epidemiology and End Results (SEER)-Medicare database, Paul et $a l$, suggested that multicentre RCTs could help to assess the usefulness of new technologies. ${ }^{21}$ Indeed, the level of evidence from studies using propensity scores is lower than that from RCTs. ${ }^{22}$ Moreover, a medicoeconomic study is needed to determine whether the costs induced by VATS are offset by the reduction in postoperative complications.
Many studies have shown that long-term survival and locoregional recurrence in patients operated on by VATS were comparable to those in patients who underwent lobectomy by thoracotomy. ${ }^{8-15}$

In 2013, the recommendations of the American College of Chest Physicians confirmed a significant reduction in respiratory complications in patients who had undergone VATS lobectomy. ${ }^{2}$ These recommendations are based on retrospective cohort studies and meta-analyses of non-randomised studies to show the clinical benefits of VATS. ${ }^{2}$

Regarding the economic impact, only a few retrospective cohort studies have evaluated the financial impact of VATS lobectomy when compared with open thoracotomy. These studies showed a trend towards a lower total cost of VATS when compared with thoracotomy, ${ }^{23-26}$ but other studies showed no difference between the two approaches. ${ }^{27} 28$

Studies highlighting the superiority of VATS reported that VATS was a more expensive surgical procedure, but the shorter length of hospital stay counterbalanced this additional cost and led to a lower total hospital cost compared with open thoracotomy. However, these studies were retrospective and did not take into account the cost of all the equipment such as video and endoscopic apparatus. Moreover, the evidence of the benefit of VATS should be confirmed by the ongoing VIOLET trial, which will compare VATS with open thoracotomy for NSCLC, in terms of effectiveness, cost-effectiveness and acceptability. ${ }^{29}$

The minimally invasive nature of VATS should have a positive impact on postoperative expectoration and ventilation, and the incidence of postoperative respiratory complications, including atelectasis, pneumonia and ARDS, should therefore be reduced by VATS. These respiratory complications are responsible for prolonged stays in ICUs and overall hospitalisation. They also have an impact on recovery and quality of life when patients return home. The reduction in the incidence of complications should counterbalance the additional cost of VATS. This paper presents a large RCT to assess the medicoeconomic impact of VATS lobectomy when compared with open thoracotomy.

\section{Objectives}

The primary aim is to evaluate the medicoeconomic impact of VATS lobectomy when compared with thoracotomy for the management of NSCLC from the societal perspective.

Secondary aims are to assess the impact of VATS on the budget to determine whether VATS is economically sustainable for the healthcare payer and for the healthcare facility, the rate of conversions from VATS to thoracotomy, postoperative complications at 30 days, postoperative death, the use of analgesics in the postoperative period, the number of mediastinal lymph nodes removed and the duration of hospital stay. Finally, other secondary aims are to evaluate quality of life at 30 days and after 3, 6 and 12 months. Long-term survival and locoregional recurrence will also be analysed. 


\section{Trial design}

The study design is a two-arm parallel RCT comparing lobectomy performed by VATS with lobectomy using thoracotomy for the treatment of lung cancer.

\section{METHODS}

\section{Participants, interventions and outcomes}

\section{Study setting}

This is a multicentre RCT involving 12 French departments of thoracic surgery. Every participating centre had already performed more than 50 VATS lobectomies.

\section{Eligibility criteria}

Inclusion criteria:

- Patients who have given their consent,

- Any patient with proven or suspected NSCLC which could be treated by lobectomy,

- Patients with a negative mediastinoscopy or negative endobronchial ultrasound following a positron emission tomography scan showing uptake in mediastinal lymph nodes in the preoperative examination,

- Age $>18$ years,

- Patients with national health insurance cover,

- Patients fit for surgical resection.

\section{Exclusion criteria:}

- Adults under wardship,

- Pregnant or breastfeeding women,

- Tumours in contact with the pulmonary artery or developing in the lobar bronchi after bronchial fibroscopy,

- Tumours invading:

- The chest wall,

- the mediastinal pleura or structures of the mediastinum (superior vena cava, trachea, the main-stem bronchi, aorta and oesophagus, vertebrae),

the diaphragm,

- the neurovascular structures of the apex (brachial plexus, subclavicular artery, subclavicular vein) causing Pancoast-Tobias syndrome,

- Patients with histologically proven contralateral or supraclavicular lymph node (N3) involvement whatever the harvesting method,

- Patients with metastasis (brain, bone, liver, adrenal glands, contralateral lung and pleura),

- Patients who have undergone neoadjuvant chemotherapy and/or radiotherapy,

- Patients who have already undergone thoracotomy,

- Patients with decompensated heart failure or with a systolic ejection fraction $<30 \%$,

- Patients with severe pulmonary artery hypertension,

- Patients with untreated valve disease,

- Patients with unstable angina despite appropriate treatment,
Patients with untreated carotid stenosis greater than $70 \%$,

- Patients with histologically proven cirrhosis with various decompensations or who have presented haemoptysis because of oesophageal varicose veins,

- Patients with severe neurological sequelae (hemiplegia, paraplegia and tetraplegia),

- Patients presenting severe psychiatric disorders (dementia, psychosis).

\section{Interventions Subtitle "interventions" is in the same paragraph as eligibility criteria}

\section{Lobectomy using video-thoracoscopy}

Two approaches are possible: either the surgeon stands behind the patient (posterior approach) or in front of the patient (anterior approach).

The operation will be performed using either approach at the discretion of each team depending on its habits:

- Either using total VATS, with three or four ports; the surgical specimen will be removed by enlarging one of the port wounds;

- Or the operation will be performed using two or three ports associated with a utility minithoracotomy of approximately $4 \mathrm{~cm}$ in the fourth or fifth intercostal space along the anterior or posterior axillary line through which the instruments will be inserted into the thorax. For the utility minithoracotomy, it is possible to use a skin retractor, but the ribs must never be spread using a Finochietto-type or Tuffiertype rib spreader. The surgeon will perform the whole operation by looking at the screen only.

The camera used will have a $10 \mathrm{~mm}$ fibrescope preferably with a visual angle of $30^{\circ}$. It will be positioned in the seventh or eighth intercostal space via a port. The specific instruments for VATS will be used, as well as an 'EndoGIA' stapler. The vascular elements of the target lobe will be dissected and then individually stapled using an EndoGIA stapler. The lobar bronchi will be dissected and individually stapled.

The surgeon must send the different lymph nodes fresh in separate flasks marked with the name of the patient, the date of the operation and the lymph node station with its corresponding number. ${ }^{28}$

Mediastinal lymphadenectomy will be performed systematically during every lobectomy. ${ }^{28}$ On the right side, it will include dissection of the superior mediastinal nodes (no 2 and no 4) and, when necessary, the prevascular lymph nodes on the anterior border of the superior vena cava and the retrotracheal nodes will be resected (no 3) and the subcarinal or tracheobronchial lymph nodes will be dissected (no 7). All of the lymph nodes in contact with the oesophagus from the arch of the azygos vein to the costophrenic angle will be resected (no 8). Finally, the pulmonary ligament will be cut to its contact with the inferior pulmonary vein and all of the lymph nodes at this station will be resected (no 9). 
On the left side, lymph nodes in the para-aortic station (no 6) and the subaortic station will be dissected (no 5). Dissection of the inferior mediastinal station will include subcarinal or tracheobronchial lymph nodes (no 7), lymph nodes in contact with the oesophagus (no 8) and pulmonary ligament lymph nodes (no 9).

The chest cavity will be drained in accordance with the habits and principles of each surgical team using one or two chest tubes. An outpatient drainage system can be used.

\section{Lobectomy using thoracotomy}

Two types of thoracotomy are possible: posterolateral thoracotomy with muscle sparing or lateral thoracotomy. Each surgical team will choose one of the two approaches in accordance with its experience. At the start of the study, each team must determine the approach it will use throughout the trial. The lobectomy will be performed in accordance with the usual principles of each team.

The surgeon must send the different lymph nodes fresh in separate flasks marked with the name of the patient, the date of the operation and the lymph node station with its corresponding number. ${ }^{28}$

Mediastinal lymphadenectomy will be performed systematically during every lobectomy as described above. ${ }^{28}$ The chest cavity will be drained in accordance with the habits and principles of each surgical team using one or two chest tubes. An outpatient drainage system can be used.

\section{Postoperative analgesia}

- Analgesia (morphine) will be delivered via the epidural catheter or paravertebral catheter or intravenously using a syringe pump self-monitored by the patient.

- For each centre, the mode of administration of analgesia will be the same for both groups. Each centre must choose a mode of administration to use throughout the whole study and up to the last inclusion.

- The combination with other analgesics is permitted.

\section{Chest physiotherapy}

All patients will have respiratory and motor physiotherapy immediately after the surgery at least twice a day during hospital stay.

\section{Oxygen therapy}

- Patients will have nasal cannula. The oxygen sensor will be removed as soon as the oxygen saturation is above $93 \%$.

- Saline aerosols will be prescribed if the patient has difficulty in expectorating.

Non-invasive ventilation (NIV) is not prescribed as a preventive therapy (unauthorised) postoperatively and for the two randomised groups. It will be used only when the patient has an element of the primary outcome (respiratory complications: severe dyspnoea or obstruction).
Thrombophlebitis will be prevented by stockings and anticoagulants. Because of the absence of consensus, postoperative anticoagulation will be managed according to the usual practices of each centre. Immediately after the surgery, the patient could be seated and then walk around the room and in the hallway.

\section{Removal of chest tubes}

The following criteria have to be respected:

- Radiography: lung to the wall and no pleural effusion.

- No bubbling cough.

- Amount of liquid $<300 \mathrm{~mL} / 24$ hours.

\section{Leaving the hospital}

- The patient can leave the hospital when the chest tube is removed.

- For patients with prolonged bubbling, the use of a Heimlich valve can be considered for the return to home.

\section{Outcomes}

\section{Primary outcomes}

An incremental cost-effectiveness ratio associated with the use of VATS when compared with thoracotomy will be estimated from the societal perspective and expressed in terms of cost per life year gained. To evaluate this final end point, an intermediate criterion will be estimated: postoperative respiratory complications. Postoperative respiratory complications include at least one of the following elements collected at 30 days:

- The use of NIV. Severe dyspnoea associated or not with bronchial congestion requiring NIV (a respiratory rate $>30$ cycles/min or with a decrease in $\mathrm{SaO}_{2}<93 \%$ ). NIV can be implemented in the surgery unit or the patient can be transferred to the ICU depending on his/her clinical status and the organisation of each centre,

- The onset of atelectasis defined as 'any congestion or obstruction of a lobe or a lung requiring one or several postoperative fibroscopic aspirations',

- Pneumonia defined by the presence of fever $\geq 38.5^{\circ} \mathrm{C}$ associated with purulent expectoration and/or associated with new infiltrate on the lung X-ray,

- Mechanical ventilation for more than 2 days after the surgery,

- Reintubation,

- ARDS defined as 'a type of lung failure that may result from any disease that causes large amounts of fluid to collect in the lungs',

- Health-related quality of life will be evaluated using the EQ-5D. ${ }^{29}$

\section{Secondary outcomes}

- Cost of the surgical technique, induced costs and avoided costs,

- Conversion from VATS to thoracotomy, 
- Intraoperative complications: including vascular injury, stapling defects (vessels or bronchi),

- Haemorrhagic complications defined as 'any intraoperative or postoperative bleeding requiring transfusions and/or a repeat operation to achieve haemostasis or to remove clots',

- Postoperative complications at 30 days:

- Prolonged air leaks, defined as 'any bubbling requiring maintenance of the chest drain beyond the $7^{\text {th }}$ day after the pulmonary resection',

- Cardiovascular complications defined as 'rhythm disorders, heart failure or cardiogenic shock, myocardial infarction, pulmonary embolism, cerebrovascular accident, acute lower-limb ischemia',

- Bronchopleural fistula diagnosed by bronchial fibroscopy,

- The total quantity (in mg) of morphine administered in the postoperative period,

- In-hospital mortality rate defined as any death occurring within the 30 days following or during the same hospitalisation as the surgery. The causes of death will be recorded, notably those related to a respiratory complication and those due to another cause,

- Duration of hospital stay (days),

- Rehospitalisation,

- The number of lymph nodes harvested from each mediastinal site will be estimated and the pTNM stage will be determined,

- Quality of life at 3,6 and 12 months using the SF-36 questionnaire.

- Survival, locoregional recurrences and adjuvant therapy use at 12, 24 and 36 months.
Participant timeline

Duration of the study

Duration will be:

- 24 months for inclusion,

- 36 months of follow-up for each patient,

- 60 months for total duration of the study (from the first inclusion to the last follow-up of the last patient).

\section{Study programme}

The study programme is summarised in table 1 .

Flow chart

The flow chart of the protocol is summarised in figure 1.

\section{Sample size}

The different cohort studies and the French thoracic surgery database Epithor report a rate of respiratory complications following lobectomy using thoracotomy of around $15 \%$. The hypothesis that lobectomy using VATS will reduce postoperative respiratory complications by $7 \%$ is seemingly pessimistic given the different North-American studies. Cohort studies report a decrease of $10 \%$ in postoperative complications using VATS. This study is a superiority trial to demonstrate that VATS will reduce respiratory complications compared with thoracotomy.

With a unilateral type I error of $5 \%$ and a type II error of $20 \%$, the estimated number of patients in each group is 256. To take into account deviations from the protocol notably conversions from VATS to thoracotomy, we wish to include 300 patients in each arm, that is to say a total of 600 patients.

\section{Recruitment}

Every centre participating in this study must include 50 patients, which means two to three patients every month.

Table 1 Summary of the study programme

\begin{tabular}{|c|c|c|c|c|c|c|c|}
\hline & Preoperative & Day 1 & Day 30 & Month 3 & Month 6 & Month 12 & $\begin{array}{l}\text { Months 24, } \\
36 \text { and } 60\end{array}$ \\
\hline Eligibility & $\sqrt{ }$ & & & & & & \\
\hline $\begin{array}{l}\text { Informed } \\
\text { consent }\end{array}$ & $\sqrt{ }$ & & & & & & \\
\hline \multicolumn{8}{|c|}{ Randomisation $\sqrt{ }$} \\
\hline \multicolumn{8}{|c|}{$\begin{array}{l}\text { Characteristics } \sqrt{ } \\
\text { of patients }\end{array}$} \\
\hline $\begin{array}{l}\text { EQ-5D } \\
\text { questionnaire }\end{array}$ & $\sqrt{ }$ & $\sqrt{ }$ & $\sqrt{ }$ & $\sqrt{ }$ & $\sqrt{ }$ & $\sqrt{ }$ & \\
\hline $\begin{array}{l}\text { SF-36 } \\
\text { questionnaire }\end{array}$ & $\sqrt{ }$ & $\sqrt{ }$ & $\sqrt{ }$ & $\sqrt{ }$ & $\sqrt{ }$ & $\sqrt{ }$ & \\
\hline $\begin{array}{l}\text { Judgement } \\
\text { criteria }\end{array}$ & & & $\sqrt{ }$ & & & & \\
\hline $\begin{array}{l}\text { Adverse } \\
\text { events }\end{array}$ & & $\sqrt{ }$ & $\sqrt{ }$ & & & & \\
\hline $\begin{array}{l}\text { Long-term } \\
\text { survival }\end{array}$ & & & & & $\sqrt{ }$ & $\sqrt{ }$ & $\sqrt{ }$ \\
\hline
\end{tabular}


Figure 1

Flow chart

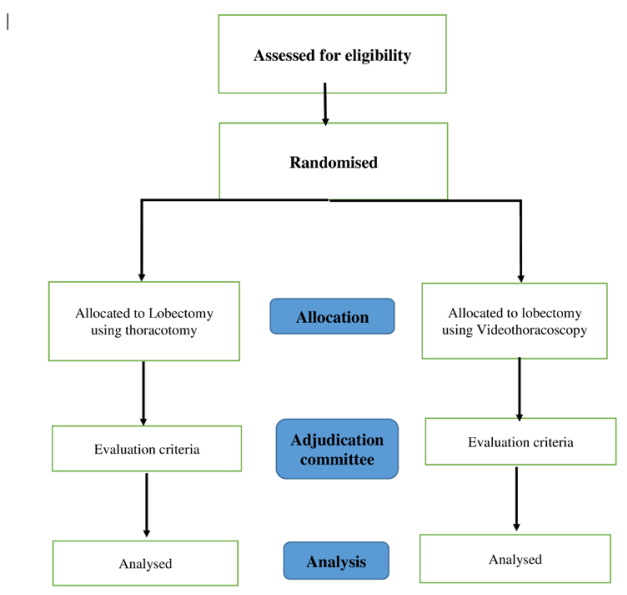

Figure 1 Flow chart of the protocol.

\section{Allocation of interventions \\ Sequence generation}

Randomisation is directly available to the investigators in the eCRF software (http://www.ecrf-medsharing.com/). The number of patients in each of the treatment groups will be equal with a ratio of 1:1. Stratification by centre is envisaged since the different practices of each team may have an impact on the judgement criterion. The list of randomised patients will be divided into blocks of 12 to obtain balanced groups. The randomisation will be available after checking inclusion criteria.

A comprehensive document describing the randomisation procedure will be kept in a confidential manner at the Clinical Research Unit of Dijon University Hospital.

\section{Blinding}

The independent adjudication committee (IAC)

\section{Members}

Three independent surgeons will constitute the IAC.

Role

Validation of the primary end point blindly.

Checking the cause of premature death (before 30 days).

\section{Method}

The patients' medical records will be completely anonymous. Checking will be done blindly: members of the IAC will not have any information on the type of procedure performed, the surgery report will not be provided nor will any documents allowing identification of the kind of surgery performed. The study centre will also be blinded. In the absence of a consensus, a telephone meeting will be organised between the three members of the IAC.

\section{Medical record provided}

ICU report and hospitalisation unit report: All postoperative follow-up records containing information about patients' clinical status, prescriptions and care delivered (NIV, prescription for antibiotics, fibroscopy examination report and intubation use).

Biological examination: white blood cells, platelets, $\mathrm{PaO}_{2}, \mathrm{PaCO}_{2}$ and $\mathrm{SaO}_{2}$.

Bacteriological examinations: expectoration and/or blood.

Pulmonary X-ray and thoracic CT scan reports.

\section{Process}

After a complete review of all documents provided by the investigation centre, experts will have to fill out a dedicated form and give an opinion about the following issues:

- Did respiratory failure require non-invasive ventilation?

- Was the patient intubated for respiratory failure?

- Did the patient develop atelectasis?

- Did the patient develop pneumonia?

- Was it ARDS?

The experts will have to answer the questions with 'Yes' or 'No'. If necessary, they are able to ask for complementary information about the clinical status of the patient and to justify their answer.

The completed form will be sent to the physician responsible for the methodology and statistics of the project, who will validate the primary end point.

Role of the methodologist:

Validate the primary end point with regard to the IAC answers. In case of disagreement in expert opinions, the majority opinion will be used. If necessary, the methodologist can organise telephone meetings with the three IAC members to clarify their position.

\section{Data management and analysis}

Data management

Only the patient's initials (first letter of the last name and the first name) are reported in the eCRF software and an anonymous code is created by the software. The code includes the patient's initials, the number of the centre and the number corresponding to the position in the list of inclusions.

Data will be collected in an electronic CRF created using CleanWeb software. It will be directly available to the investigators online at the following address: https:// chu-dijon.tentelemed.com/Ctms-chud/portal/login. After identification using a login and a password created at the Clinical Research Unit of Dijon University Hospital, a comprehensive document describing the patient inclusion procedure will be provided to the investigators. Investigators are responsible for the accuracy, quality and 
relevance of all data entered. These data are immediately verified through edit checks. As such, any changes in values in the CRF must be validated. These changes will be the subject of an audit trail. Justification for changes can be included. The printing of the eCRF will be requested at the end of study.

\section{Statistical methods}

All of the patients included will remain in their randomisation group as it is an intention-to-treat study. The two treatment groups will be compared in terms of patients' characteristics: age, sex, lung function tests, performance status, body mass index, comorbidities and TNM stage. Qualitative variables will be compared using the $\chi^{2}$ test (or Fisher's test if the expected values are $<5$ ) and continuous variables by Student's t-test (or the Mann-Whitney U test if the assumptions of normality or homoscedasticity are not verified).

These comparisons will check the quality of randomisation.

\section{Principal judgement criteria}

A global analysis of the composite end point will be achieved and expressed as the percentage and relative risk with their $95 \%$ CIs. For the comparison of the two treatment groups without statistical adjustment, a $\chi^{2}$ test will be used. For the comparison of the two treatment groups with statistical adjustment, a logistic regression model will be used. The statistical adjustment will be performed by introducing the following variables into the model: age, sex, forced expiratory volume, the body mass index, comorbidities and TNM stage. The adequacy of the model will be verified by the Hosmer-Lemeshow test. For the estimation of the SD of the coefficients of the logistic model, we will take into account the centre's effect ('standard error robust clustering').

A further analysis will be conducted for each item of the composite end point in order to verify the weight of each of them on the overall result. This additional analysis will only be exploratory without a causal interpretation.

\section{Secondary judgement criteria}

For the comparison of dichotomous and categorical variables, a $\chi^{2}$ test will be used. For the comparison of continuous variables, such as holding time of the operating room, duration of ICU stay, the amount of morphine administered and length of hospital stay, a Student's t-test will be used.

Survival rates will be calculated using the Kaplan-Meier method. Postoperative deaths will be taken into account in the calculation of the survival rates. The comparison of the survival curves will be computed using the log-rank test. A proportional hazards model (Cox model) will be used to adjust the comparison of the two groups for prognostic factors for survival.

For the analysis of the quality of life scores, an analysis of variance for repeated measures will be used to model time effects and to test the treatment effect with regard to the interaction time. No intermediate analyses are planned in the protocol. The threshold for significance is set at 0.05 . The analysis will be provided by the Clinical Research Unit of Dijon University Hospital.

\section{Monitoring}

\section{Data monitoring}

A clinical research assistant, delegated by the promoter (clinical research assistant (CRA) promoter), will regularly visit every centre involved in the study during the implementation of the trial, one or several times during the trial according to a predetermined frequency depending on the level of risk attributed to the research.

The aims of these visits are to:

- make sure that the protocol is followed,

- check that informed consent has been given,

- check the reporting of serious adverse events,

- follow the traceability of study drugs (visits to the pharmacy, storage of and records for drugs),

- ensure quality control: compare data in the eCRF with those in the source documents of the centre.

Persons in charge of quality control for biomedical research and duly mandated by the promoter to do this have access, provided that the persons concerned have agreed, to participants' individual data that are strictly necessary for this control. They are subject to professional secrecy in the conditions defined by articles 226-13 and 226-14 of the penal code.

After every visit, a written monitoring report will be provided (traceability of visits).

Harm

An adverse event is defined as 'any untoward medical occurrence in a patient or clinical trial subject enrolled in a biomedical research whether or not this symptom is related to the research or the product used' or 'an event which is related to the research or the product used'.

A serious adverse event is defined as 'any untoward medical occurrence that at any dose fulfils at least one of the following criteria':

- Is fatal (results in death; NOTE: death is an outcome, not an event),

- Is life threatening (NOTE: the term 'life threatening' refers to an event in which the patient was at risk of death at the time of the event; it does not refer to an event which could hypothetically have caused death if it were more severe):

- Requires inpatient hospitalisation or prolongation of existing hospitalisation,

- Results in persistent or significant disability/ incapacity (The terms 'disability' and 'incapacity' mean any clinically significant temporary or permanent physical or mental disability that affects the physical activity and/or quality of life of patients),

In the context of this protocol, the following will be considered serious adverse events: 
- any event that leads to the death or threatens the life of the person taking part in the research,

- any event that requires hospitalisation or prolongation of the hospitalisation,

- any event that causes severe or lasting disability or handicap,

Note: in case of failure of medical devices used for VATS or thoracotomy, it should be reported as usual to local medical-device surveillance correspondents. Moreover, if the failure causes a serious adverse event, in addition to reporting to medical-device surveillance correspondents, the investigator must fill in a serious adverse event form and send it to the sponsor.

Description of expected adverse events

Expected adverse events/effects are:

- adverse events related to anaesthesia,

- perioperative complications: haemorrhage, arrhythmia, vascular injury, stapling defects,

- postoperative complications:

- respiratory complications: bronchial obstruction, pleural effusion, pneumonia, atelectasis, ARDS, prolonged air leaks, torsion of lobe, pulmonary infarct, chylothorax,

- infectious complications: pyothorax, postprocedural site wound infection, bronchopneumopathy, pulmonary empyema,

- haemorrhagic complications,

- cardiovascular complications: pulmonary oedema, cardiac failure, arrhythmia, myocardial ischaemia, acute lower-limb ischaemia, cerebrovascular accident, cardiogenic shock,

- postoperative pain,

- bronchopleural fistula,

- venous thromboembolic events,

- subcutaneous emphysema,

- nerve injury (including recurrent nerve paralysis, phrenic nerve paralysis, intercostal nerve injury, brachial plexus lesion),

- rib fracture.

Conduct in case of such events

The investigator will make the initial declaration of a serious adverse event. The principal investigator informs the sponsor by telephone, fax or mail of the onset of any serious adverse event (that is to say whether $t$ the event is linked to the product being studied or to the research), whether it is expected or unexpected. Adverse events that are not serious will be recorded in the case report forms.

This information must be passed on within 24 working hours following discovery of the serious adverse event, and then by mail within 48 working hours, by sending the declaration form for serious adverse events completed and signed by an investigator to:

CHU Dijon-Direction de la Recherche Clinique, 14 Rue Gaffarel, 21079 Dijon cedex

Fax: 03.80.29.36.90

\section{Auditing}

The investigators accept to meet the requirements of the promoter and the competent authority concerning study audits or inspections. Audits can take place at any stage of the study, from the development of the protocol to publication of the results and archiving of the data used or produced in the context of the study.

\section{Ethics and dissemination}

\section{Research ethics approval}

The trial cannot begin until the authorisation of both the Comié de Protection des Personnes (CPP) and the Competent Authority (French Medicines Agency) has been obtained. The protocol was approved by the CPP Est I the 9 June 2015, and authorisation was obtained from the French Medicines Agency on the 9 June 2015.

The authorisation of the competent authority becomes null and void if within 6 months following the authorisation the research has not started (meaning no one has been included in the protocol). The authorisation of the CPP becomes null and void if within 1 year following approval the research has not started (meaning no one has been included in the protocol).

Neither the investigator nor the promoter can modify this protocol without the prior written agreement of the other party. If substantial modifications have to be made, they must be made via an amendment to the protocol. This amendment will be applied once it has been authorised by both the CPP Est III and the competent authority.

\section{Consent or assent}

The surgeon will inform patients of the existence of the study during the preoperative consultation and will ask for their agreement to participate in the study after checking the inclusion and non-inclusion criteria.

In the preoperative period, patients' characteristics will be collected (age, sex, WHO performance status, body mass index, comorbidity and TNM stage). Pulmonary function tests, blood gas tests and lung cancer check-ups will be done as usual.

\section{Confidentiality}

In accordance with the provisions of article R. 5120 of the Public Health Code, the investigator and any person involved in the trial will be bound by professional secrecy, in particular with regard to the nature of the products being studied, the trials, the persons taking part and the results obtained subject to the provisions set out in article L. 1123-9 (new numbering system) of the Public Health Code. Unless they have the agreement of the promoter (Dijon CHU), they can only provide information on the study to the Health Authorities, including inspectors as mentioned in article L.209-13 (old numbering system) of the Public Health Code.

No comments about the trial, either verbally or in writing, will be made without the authorisation of both the coordinating investigator and the promoter (Dijon CHU). 
The computer file used for this research will require the authorisation of the 'Commission Nationale de l'Informatique et des Libertés' in application of articles 40-1 and in accordance with the law 'Computer science and Freedom'. Patients' medical data will be processed by computer and will only be communicated to the promoter and, if needed, to the Health Authorities, in conditions that ensure their confidentiality. Patients can exercise their right to have access to and to rectify their data by contacting the investigator.

\section{Dissemination policy}

Dijon University Hospital is interested in the publication of the results of every trial. For every publication, the investigator must mention Dijon University Hospital, in accordance with the Charter for Publications.

In studies funded by the PHRC or by specific funding provided by Dijon University Hospital (eg, internal call for projects), the publication must specify the sources of funding, the promoter.

The results of this trial will be submitted to a journal related to thoracic surgery.
Author affiliations
${ }^{1}$ Department of Thoracic and Cardiovascular Surgery, CHU Dijon, Dijon, France
${ }^{2}$ Department of Health Economy, CHU Dijon, Dijon, France
${ }^{3}$ Department of Epidemiology, CHU Dijon, Dijon, France
${ }^{4}$ Department of Thoracic Surgery, AP-HP, Hôpital Cochin, Paris, France
${ }^{5}$ Department of Thoracic Surgery, CHU Rouen, Rouen, France
${ }^{6}$ Department of Thoracic and Cardiovascular Surgery, Centre Jean Perrin, Clermont- Ferrand, France
${ }^{7}$ Department of Thoracic and Cardiovascular Surgery, CHU Rennes, Rennes, France ${ }^{8}$ Department of Thoracic and Vascular Surgery, Hôpital Tenon, AP-HP, Paris, France ${ }^{9}$ Department of Thoracic Surgery, HCL, Hôpital Louis Pradel, Bron, France ${ }^{10}$ Department of Thoracic and Cardiovascular Surgery, CHU Poitiers, Poitiers, France ${ }^{11}$ Department of Thoracic and Cardiovascular Surgery, Hôpital Pasteur, CHU Nice, Nice, France
${ }^{12}$ Department of Thoracic Surgery and Diseases of Oesophagus, Assistance Publique des Hôpitaux de Marseille, North Hospital, Marseille, France
${ }^{13}$ Department of Thoracic Surgery, Hôpital Civil, CHU Strasbourg, Strasbourg, France ${ }^{14}$ Department of Thoracic and Cardiovascular Surgery, Hôpital Arnaud de Villeneuve, CHU Montpellier, Montpellier, France

Contributors All authors contributed to the design of this trial protocol. $A B$ is the chief investigator for Lungsc001 and leads the project. PBP was responsible for the initial development of the protocol along with AB. PBP and HAH coordinated development of trial documentation and took main responsibility for preparing and editing this article for journal publication. ACB is responsible for the medicoeconomic analysis, and LSA coordinates the methodology. All authors read and approved the final manuscript.

Competing interests Jean-Marc Baste reports consulting and lecture fees from Covidien and consulting fee from Ethicon. The other authors have nothing to disclose with regard to commercial support.

Ethics approval The protocol was approved by the 'Comité de Protection des Personnes' Est 1 on the 9th of June 2015, and authorisation was obtained from the French Medicines Agency on the 9th of June 2015.

Provenance and peer review Not commissioned; externally peer reviewed.

Open Access This is an Open Access article distributed in accordance with the Creative Commons Attribution Non Commercial (CC BY-NC 4.0) license, which permits others to distribute, remix, adapt, build upon this work non-commercially, and license their derivative works on different terms, provided the original work is properly cited and the use is non-commercial. See: http://creativecommons.org/ licenses/by-nc/4.0/ (c) Article author(s) (or their employer(s) unless otherwise stated in the text of the article) 2017. All rights reserved. No commercial use is permitted unless otherwise expressly granted.

\section{REFERENCES}

1. Binder-Foucard F, Bossard N, Delafosse P, et al. French network of cancer registries (Francim). Cancer incidence and mortality in France over the 1980-2012 period: solid tumors. Rev Epidemiol Sante Publique 2014;62:95-108.

2. Howington JA, Blum MG, Chang AC, et al. Treatment of stage I and II non-small cell lung cancer: Diagnosis and management of lung cancer. Chest 2013(143):e278S-313.

3. Paul S, Altorki NK, Sheng S, et al. Thoracoscopic lobectomy is associated with lower morbidity than open lobectomy: a propensitymatched analysis from the STS database. J Thorac Cardiovasc Surg 2010;139:366-78.

4. Thomas PA, Berbis J, Falcoz PE, et al. EPITHOR Group. National perioperative outcomes of pulmonary lobectomy for cancer: the influence of nutritional status. Eur J Cardiothorac Surg 2014;45:652-9.

5. Stéphan F, Boucheseiche S, Hollande J, et al. Pulmonary complications following lung resection: a comprehensive analysis of incidence and possible risk factors. Chest 2000;118:1263-70.

6. Pagès PB, Delpy JP, Orsini B, et al. Propensity score analysis comparing videothoracoscopic lobectomy with Thoracotomy: a French Nationwide Study. Ann Thorac Surg 2016;101:1370-8.

7. Bendixen $\mathrm{M}$, Jørgensen OD, Kronborg C, et al. Postoperative pain and quality of life after lobectomy via video-assisted thoracoscopic surgery or anterolateral thoracotomy for early stage lung Cancer: a randomised controlled trial. Lancet Oncol 2016;17:836-44.

8 Whitson BA, GrothSS, Duval SJ, et al. Surgery for early-stage non-small cell lung cancer: a systematic review of the videoassisted thoracoscopic surgery versus thoracotomy approaches to lobectomy.Ann Thorac Surg2008 88200816

9. Yan TD, Black D, Bannon PG, et al. Systematic review and metaanalysis of randomized and nonrandomized trials on safety and efficacy of video-assisted thoracic surgery lobectomy for early-stage non-small-cell lung Cancer. J Clin Oncol 2009;27:2553-62.

10. Li Z, Liu H, Li L. Video-assisted thoracoscopic surgery versus open lobectomy for stage I lung cancer: A meta-analysis of long-term outcomes. Exp Ther Med 2012;3:886-92.

11. Zhang $\mathrm{Z}$, Zhang $\mathrm{Y}$, Feng $\mathrm{H}$, et al. Is video-assisted thoracic surgery lobectomy better than thoracotomy for early-stage non-smallcell lung Cancer? A systematic review and meta-analysis. Eur J Cardiothorac Surg 2013;44:407-14.

12. Cao C, Manganas C, Ang SC, et al. A meta-analysis of unmatched and matched patients comparing video-assisted thoracoscopic lobectomy and conventional open lobectomy. Ann Cardiothorac Surg 2012;1:16-23.

13. Taioli E, Lee DS, Lesser M, et al. Long-term survival in videoassisted thoracoscopic lobectomy vs open lobectomy in lung-cancer patients: a meta-analysis. Eur J Cardiothorac Surg 2013;44:591-7.

14. Chen FF, Zhang D, Wang YL, et al. Video-assisted thoracoscopic surgery lobectomy versus open lobectomy in patients with clinical stage I non-small cell lung cancer: a meta-analysis. Eur J Surg Oncol 2013;39:957-63.

15. Cai YX, Fu XN, Xu QZ, et al. Thoracoscopic lobectomy versus open lobectomy in stage I non-small cell lung Cancer: a meta-analysis. PLoS One 2013;8:e82366.

16. Kirby TJ, Mack MJ, Landreneau RJ, et al. Lobectomy--videoassisted thoracic surgery versus muscle-sparing thoracotomy. A randomized trial. J Thorac Cardiovasc Surg 1995;109:997-1002.

17. Sugi K, Kaneda Y, Esato K. Video-assisted thoracoscopic lobectomy achieves a satisfactory long-term prognosis in patients with clinical stage IA lung cancer. World J Surg 2000;24:27-31.

18. Palade E, Passlick B, Osei-Agyemang T, et al. Video-assisted vs open mediastinal lymphadenectomy for Stage I non-small-cell lung cancer: results of a prospective randomized trial. European Journal of Cardio-Thoracic Surgery 2013;44:244-9.

19. Shigemura N, Akashi A, Nakagiri T, et al. Complete versus assisted thoracoscopic approach: a prospective randomized trial comparing a variety of video-assisted thoracoscopic lobectomy techniques. Surg Endosc 2004;18:1492-7.

20. Craig SR, Leaver HA, Yap PL, et al. Acute phase responses following minimal access and conventional thoracic surgery. Eur $J$ Cardiothorac Surg 2001;20:455-63.

21. Paul S, Isaacs AJ, Treasure T, et al. Long term survival with thoracoscopic versus open lobectomy: propensity matched 
comparative analysis using SEER-Medicare database. $B M J$ 2014;349:g5575.

22. Heinze G, Jüni P. An overview of the objectives of and the approaches to propensity score analyses. Eur Heart $J$ 2011;32:1704-8.

23. Cho S, Do YW, Lee EB. Comparison of costs for video-assisted thoracic surgery lobectomy and open lobectomy for non-small cell lung Cancer. Surg Endosc 2011;25:1054-61.

24. Swanson SJ, Meyers BF, Gunnarsson CL, et al. Video-assisted thoracoscopic lobectomy is less costly and morbid than open lobectomy: a retrospective multiinstitutional database analysis. Ann Thorac Surg 2012;93:1027-32.

25. Casali G, Walker WS. Video-assisted thoracic surgery lobectomy: can we afford it? Eur J Cardiothorac Surg 2009;35:423-8.

26. Farjah F, Backhus LM, Varghese TK, et al. Ninety-day costs of videoassisted thoracic surgery versus open lobectomy for lung cancer. Ann Thorac Surg 2014;98:191-6.

27. Park BJ, Flores RM. Cost comparison of robotic, video-assisted thoracic surgery and thoracotomy approaches to pulmonary lobectomy. Thorac Surg Clin 2008;18:297-300.
28. Deen SA, Wilson JL, Wilshire CL, et al. Defining the cost of care for lobectomy and segmentectomy: a comparison of open, videoassisted thoracoscopic, and robotic approaches. Ann Thorac Surg 2014;97:1000-7 http://www.hra.nhs.uk/news/research-summaries/ the-violet-study/.

29. Lim E. VIdeo assisted thoracoscopic lobectomy versus conventional Open LobEcTomy for lung cancer, a multi-centre randomised controlled trial with an internal pilot . 2015 http://www.hra.nhs.uk/ news/research-summaries/the-violet-study/.

30. Rusch VW, Crowley J, Giroux DJ, et al. International Staging CommitteeCancer Research and BiostatisticsObservers to the CommitteeParticipating Institutions. The IASLC Lung Cancer Staging Project: proposals for the revision of the $\mathrm{N}$ descriptors in the forthcoming seventh edition of the TNM classification for lung cancer. J Thorac Oncol 2007;2:603-12.

31. EuroQol Group. EuroQol-a new facility for the measurement of health-related quality of life. Health Policy Amst Neth 1990;16:199-208. 